\title{
The Swedish paradox explained? Investigating the role of economic inequality and risk perceptions in the Novel Coronavirus Pandemic
}

\author{
Nate Breznau ${ }^{1}$, (DD 0000-0003-4983-3137 \\ Lisa Heukamp ${ }^{1}$, (DD 0000-0002-6381-7099 \\ Hung H.V. Nguyen ${ }^{1}$, (D) 0000-0001-9496-6217 \\ ${ }^{1}$ University of Bremen, SOCIUM Research Center on Inequality and Social Policy
}

\begin{abstract}
Economic inequality is understood as a country-level risk factor that increases the spread of infectious diseases. The Novel Coronavirus presents somewhat contradictory evidence of this because the most equal countries in the world experienced deaths per capita as high as the most unequal. We label this the 'Swedish paradox'. We theorize that inequality has a non-linear association with disease spread because of risk perceptions among the public. Risk perceptions that are extreme in either direction - toward nonchalance or outright panic - lead to behaviors that increase infection(1). Highly equal countries like Sweden had very low risk perceptions at the beginning of the pandemic and this may have led to individual and public actions that increase infection risk. To test this, we modeled the role of economic inequality on infection through the mediating role of risk perceptions in April, in 74 countries. We measured the intensity of the outbreak and government interventions in March as key predictors of risk perceptions. This lets us identify 'over' or 'under' concern and whether these extremes in the distribution of risk perceptions predicts infection increase in May, measured as death rates with an 18-day lead. We find that inequality has a large linear association with risk perceptions in April ( 0.50 as a standardized [beta] coefficient). We find that risk perceptions in April have a moderate association with infection increase in May ( 0.24 beta) and these effects are non-linear. Societies below a disposable income inequality Gini of around 31 were prone to slightly more infection in May as a function of less inequality, whereas societies above 31 were prone to much more infection as a function of more inequality, all else equal. This offers some clarification of the Swedish paradox; however, the biggest effects of inequality on infection occur at the upper end of the distribution meaning inequality is only a small piece of this puzzle.
\end{abstract}


For decades, scientists watched global income inequality increase with skepticism given findings linking economic inequality with social harms(2). These findings demonstrate that people in the lower part of the income distribution in societies with higher inequality face greater social risks, for example falling into poverty, becoming unemployed, moving frequently in and out of poverty, greater degrees of health problems, greater likelihood of incarceration of both self and family members, greater likelihood of a younger or single parent head of household and greater likelihood of a lower educated parent(3-6). In addition to heightened social risks for those lower in the economic distribution, in more unequal societies the middle and upper classes are more likely to fear crime victimization, suffer work-related mental health issues, and express greater concern over inequality problems. Moreover, everyone in unequal societies tend to have less social trust and experience group conflicts along ethnic, racial, political or religious lines on average $(7,8)$.

A pandemic exacerbates these preexisting social problems stemming from inequality because it suddenly introduces new risks. These risks fall disproportionately on the lower social strata. The degree of this risk disproportionality is a function of existing economic inequality. Inequality also increases risk of infection because the greater the inequality, the more that people in the lower income strata live in higher density neighborhoods and dwellings, and work in unhealthy conditions, more often informal or quasi-formal forms of employment, where effective social distancing and quarantining are less feasible. Such strata often lack access to adequate health care which could otherwise limit the spread and reduce the death rate $(9,10)$. When any segment of the population is susceptible to higher infection rates in a pandemic, the entire population is at risk. Two recent studies confirm this by demonstrating strong statistical association of income inequality and poverty rates with COVID-19 mortality across a diverse set of countries $(11,12)$. However, inequality is not the only factor. For example, highly unequal China had some of the fewest deaths in the world, while highly equal Sweden had some of the most by May of 2020.

Government response and human behaviors are also key factors. Swift and strong lockdown measures were directly proportional to less deaths at the beginning of the COVID-19 pandemic(13). Yet government response is only as effective as the behavioral response it causes among the public. Therefore, governmental response cannot single-handedly contain the spread of a pandemic $(12,14)$. At the same time, without governmental intervention, individual behaviors are unlikely to follow best practices to prevent infection due to a lack of behavioral incentives. The evaluation of risk is key to explaining human behaviors in a pandemic(15). There are always segments of the public who do not seek information or simply misjudge the severity of the situation who will increase infection without government intervention. Where risk perceptions are too low, individuals and governments fail to take threats seriously(1),(15). When too high, panic may ensue leading to irrational and risk-seeking behaviors. 
This bring us back to the interesting case of Sweden. Early in the pandemic, per capita death rates from COVID-19 were among the highest in the world. This is a product of weak government response, but also likely a product of public under-estimation of risk. The government reaction was influenced by some Swedish pandemic experts; however, it likely reflected the preferences of the Swedish people - namely their level of concern. In highly equal societies, public concern over risks tend to be very low because of a high degree of social security, health care and social inclusion of all legal residents regardless of income or wealth. The government's response and the public's level of concerns are interrelated. This is a fundamental aspect of research on democratic governance: each causes and reinforces the other(16). In the case of Sweden, and many other highly equal societies, this produces a context of weak response and nonchalant public reaction. On the other hand, in highly unequal countries, the public are likely to panic given a lack of basic social securities like health care and unemployment protections. This level of panic may contribute to a strong governmental response, but individual behaviors may still circumvent this response and cause increased infection risks. Moreover, individuals are less likely to follow their government in highly unequal societies because of weak trust in society and government(11).

This brings us to our theoretical model of public risk perceptions as central to explaining COVID-19 infection rates, because they moderate the impact of inequality and create the possibility for too little inequality to cause higher infection rates - as in the 'Swedish paradox'. 


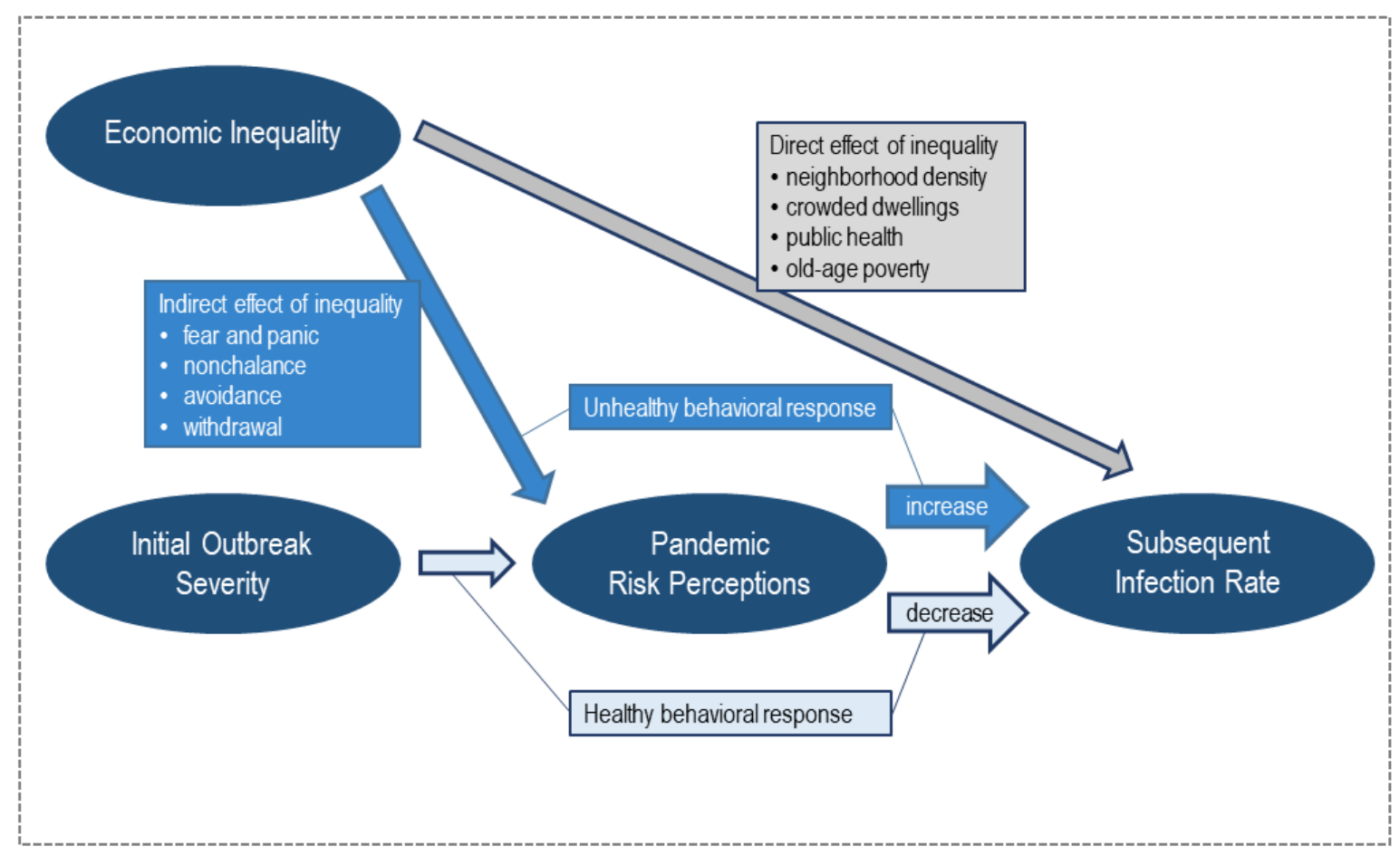

Fig 1 | Theoretical model of economic inequality, risk perceptions and COVID-19 mortality. The severity of the pandemic outbreak in a given country causes the level of risk perceptions and this impacts the subsequent infection rate. Economic inequality causes infection rates directly due to socio-economic and health factors. Economic inequality also alters risk perceptions therefore altering infection rates indirectly through risk perceptions. 


\section{Research Design}

All replication materials and supplementary results are available in the Technical Appendix (https://github.com/nbreznau/Ineq_Risk_Covid).

\section{Overview}

In March of 2020 a global team of researchers led by Andreas Lieberoth started a survey project COVIDiSTRESS (CiS), to investigate the impact of the Pandemic on individual mental health(17), thus allowing us to measure pandemic risk perceptions. The Lieberoth team made their data immediately available via the Open Science Framework. The CiS data includes a five question battery on, "Concern about the consequences of the coronavirus" for "yourself", "family", "friends", "your country" and "other countries". Respondents answer along a six-point scale from strongly disagree $(=1)$ to strongly agree $(=6)$ with a statement that they are concerned. Previous research suggests the first three questions demonstrate strong fit in a multi-group confirmatory factor analysis across 47 countries, i.e., they are probably metric invariant passing the minimum criteria for cross-national comparison $(C F A$ : $\mathrm{TLI}=0.998, \mathrm{CFI}=0.994$, RMSEA $=0.032 ; M G C F A$ : TLI $=0.992$, CFI $=0.983$, RMSEA $=0.061)(18,19)$. Following this previous research, we constructed a mean scale to measure average risk perceptions at the personal level across 74 countries as our dependent variable $($ mean $=4.504$, s.d. $=0.360)$. We excluded all countries with less than 20 respondents; however, the number of cases per country varied considerably ( mean $=1,451$; s.d. $=$ 3,237 ; range $=20$ to 21,087 ) leading to a within-country standard error of the mean ranging from 0.01 to 0.34 .

We elected to limit the CiS data to the period from March 26th to April 31st of 2020. We select this period because it marked the transition from several local outbreaks to a global pandemic in terms of deaths; it is also when the survey gathered the greatest number of respondents. On March $15^{\text {th }}$ only 14 countries experienced any deaths from the virus, but over the next month a dramatic global increase occurred and 133 countries reported deaths by April $15^{\text {th }}$. Figure 2 presents the severity of the outbreak by country and provides a visualization of the data timeframe. It presents the number of deaths with an 18day lead, as this was the average time it took COVID-19 to cause death in the early stages of the pandemic(20); values are log transformed for visualizing the 'curves'. 


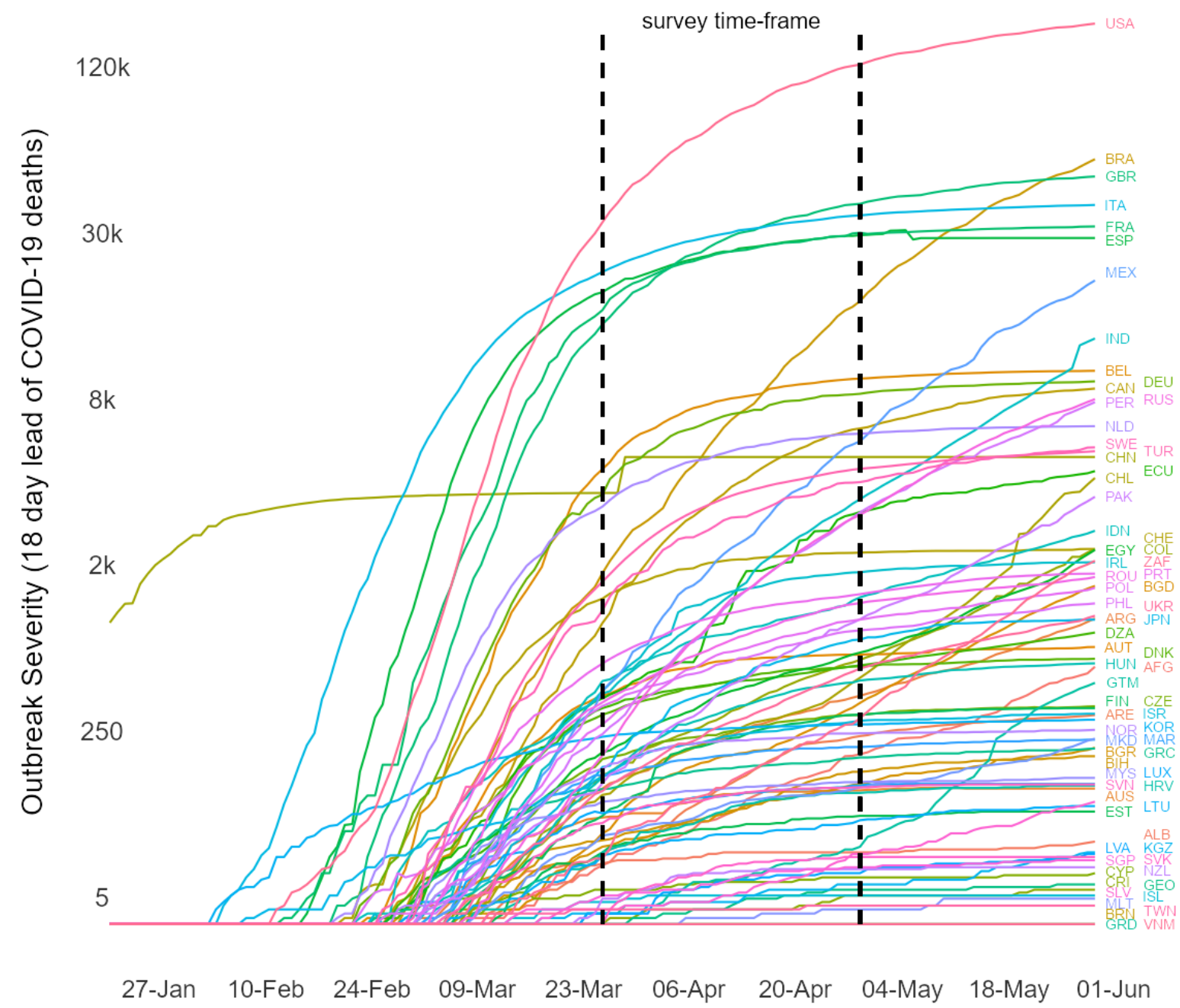

Fig 2 Severity of the COVID-19 outbreak by country in the first half of 2020. Measuring COVID-19 deaths with an 18-day lead gives a rough picture of infection rates by country as an alternative to reported positive tests because these are unreliable due to asymmetrical cross-national testing practices. Values logged for visualization purposes. In just one month from mid-May to midApril, 2020, the number of countries experiencing deaths due to COVID-19 increased from 14 to 133 , and it is likely that every country with a population over 10,000 had experienced infections by the end of April.

As shown in Figure 2, some countries such as China, Italy and Spain saw an early spike in deaths and then a 'flattening' of the curve by March, whereas others such as Russia, Mexico and Guatemala were only starting to spike in April and had no flattening. From a risk perception perspective this is crucial because humans mentally adapt to risks and continually update their assessments based on new information. Thus, Italy was ravaged by COVID-19 deaths in February but Italians were less concerned 
by April because their government took strong intervention measures and they witnessed the curve flattening, even though people were still dying. A study in Germany demonstrates this impact showing that risk perceptions decreased over time as a function of the death curve(21). At the same time, countries such as the United States and Australia saw their curves flatten but still had increases in infection, potentially re-igniting risk perceptions. As data on new confirmed cases are a result of both the infection rate and the amount of testing, these are highly unreliable as a measure of the pandemic; yet, these are the data available to the public, therefore the number of 'new cases' is the main indicator on which the public can make their risk assessments as the actual number of new cases is unknown in each country in April. This means that societies testing more are likely to have higher risk perceptions.

To attempt to account for all these factors in a statistical model without introducing too many independent variables we use days since curve inflection measured as the number of days since the number of deaths started to decrease on a week-over-week basis. Countries not yet reaching their curve inflection by the time of the survey got a score of zero. We also measure the number of new cases in the past week linearly transformed into a scale from -1 to 1 where zero is no week-over-week change and 1 or -1 are changes at the extreme in either direction. Together these two variables should measure the local outbreak severity both objectively and subjectively as available information for the public. These data come from the Johns Hopkins Coronavirus Resource Center(22). In order to measure the intensity of government intervention we take a severity scale of 17 types of prevention and lockdown measures that governments could take, developed by a team at the Blavatnik School of Government at Oxford(23). We take the value on March $15^{\text {th }}$ to allow for a lag for individuals to become aware of the measures and possibly see that they might have an impact. These data are made easily accessible via Max Roser and colleagues's Our World in Data web portal(24).

Stage 1. Does economic inequality increase risk perceptions?

In theory we expect risk perceptions to be a function of the outbreak severity plus the degree of government intervention. These factors should explain risk perceptions on average across countries; we translate this theoretical claim into a statistical model labeled "M1" in our analysis. This provides a baseline of how the local outbreak enters the public evaluation of risk. We then complement this baseline model by adding disposable income inequality as a variable to test if it explains a significant portion of the variance in risk perceptions not explained by outbreak severity and government intervention (this is M2). We measure income inequality using the Solt Standardized Income Inequality Database's 2019 averaged post-tax and post-transfer Gini coefficients(25). We use disposable income because it reflects the actual cash-in-hand of a person or family per month. The alternative is net income inequality, but this 
measure is taken before taxes and transfers and presents a very inaccurate picture of the monthly finances of individuals. We use standard ordinary least squares (OLS) regression as opposed to general least squares multilevel modeling in Stage 1 given that we have estimates only at the country-level and an outcome and test variable that are both linear. We were unable to use individual-level data as about onethird of the participants did not consent to the use of their personal information, so rather than cut cases and introduce bias and lose more country-cases, we elected to use aggregate measures from the full sample.

As economic inequality is an indicator of myriad social, economic and political asymmetries, risks, institutions and processes, it is possible that there are other variables that better explain risk perceptions; what one might call 'lurking' confounders in the causal inference language. Two in particular that worried us are the strength of the welfare state and the wealth of a given country. Therefore, we run alternative versions of M2 where we $\left(1^{\text {st }}\right)$ replace income inequality with a standardized scale combining welfare state spending as a percentage of GDP and coverage of work-related social insurance that we label welfare state strength measured from the $I L O(\mathrm{M} 3)$; and $\left(2^{\text {nd }}\right)$ use GDP per capita instead of income inequality measured using the Maddison Data for 2018 or latest year (M4). Both of these measures seemed to be influential on risk perceptions in previous research, although their specific linkages to COVID-19 deaths have not been studied to our knowledge(18). Finally, we run a model with all three alternative test variables included (M5) (M1-M5, see Tbl 1).

Mostly out of curiosity, we tested an alternative measure of income inequality as the top 1\% concentration of income using the World Inequality Database 2019 (https://wid.world/) and re-ran M1M5 with this in lieu of disposable income inequality (M11-M15, see Technical Appendix). The top 1\% variable is only a pre-tax measure so it does not account for the impact of the welfare state which is probably a big factor in the income inequality indicator; however, it does provide insight into how much the economic side of society is dominated by a small elite. We are cautious with top $1 \%$ as a variable because we only have data for 57 countries.

Stage 2. Do risk perceptions increase the spread of COVID-19?

The results of Stage 1 revealed that economic inequality has a strong statistical association with risk perceptions, therefore we proceeded with Stage 2 to test if this relationship predicts increases in infections as theorized (see Fig 1). We measure the spread of infection based on the number of deaths with an 18day lead (shown in Fig 2). We use this measure to calculate our outcome variable increase in infection ratio by subtracting its value on May $1^{\text {st }}$ from its value on May $31^{\text {st }}$ divided by the starting value on May 
$1^{\text {st }}$. Thus, a score of 1.50 would indicate that the total number of infections since the beginning of the pandemic increased by $150 \%$ in May. We then regress this outcome on predicted risk perceptions and economic inequality plus the severity of outbreak and government intervention variables. We include non-linear effects for both risk perceptions and income inequality because the second stage of our theory suggests that risk perceptions might increase infections when either too low (e.g., nonchalance, misjudging risk) or too high (e.g., irrational behaviors, fear, anger), and the same for inequality being too low (e.g., overly trusting government and others, strong sense of social security) or too high (e.g., extreme inefficiencies or corruption in government, highly competitive social norms). Therefore, we include a squared term for both variables. We present these OLS models M21-M27 (see Technical Appendix) and the same models with the alternative measure of top 1\% income concentration (M31-M37, see Technical Appendix). We run these models mostly for show, as OLS is not the most ideal method here because it cannot simultaneously account for interdependent effects across two regressions. Given that these types of models have two dependent variables - here risk perceptions ("M") and increase in infections ("Y") regressed simultaneously on a set of independent variables, we select a strategy of maximum-likelihood using structural equation modeling for our main models. We present these main models M40-M43 in Tbl 2 (and alternate M50-M53 using top 1\% in the Technical Appendix).

Estimating an impact of economic inequality indirectly through risk perceptions is a standard statistical investigation known as mediation analysis and is used to decompose statistical effects into total, direct and indirect effects(26). In our model there are non-linear variables and these translate into statistical effects that vary with the values of the independent variables. The size of an effect depends on where in the distribution of the variable one looks, analogous to a rate of change in calculus. To capture non-linear effects we follow the instantaneous indirect effects strategy described in the work of Hayes and Preacher(27).

As with any survey, there is potential random sampling variation in the CiS; however, in this case it poses a particular threat to the reliability of our tests because the sample size by country varies so widely. Therefore, we put the robustness of our findings to a test by re-running the main model (M43) on 2,000 plausible values of the country-level mean risk perceptions simulated using the within-country standard error of the mean and two random number generators. 


\section{Results}

Inequality is a major predictor of risk perceptions

Table 1 presents results of Stage 1. We find that the severity of the outbreak and government response account for about $21 \%$ of the explained variance in average risk perceptions across countries $\left(\mathrm{R}^{2}=0.206\right.$, M1). When we add income inequality it increases the explained variance to nearly $40 \%(0.392, \mathrm{M} 2)$. The coefficient for income inequality is 0.03 and significantly different from zero with greater than a $99.9 \%$ confidence interval. If M2 were an accurate model of the data generating process, we would conclude that a one-point increase in the disposable income Gini increases risk perceptions by 0.03 on a scale where the standard deviation is 0.36 . In other words, the coefficient beta is 0.50 , meaning that one standard deviation increase in Gini (about 6 points) leads to a half standard deviation increase in risk perceptions (about 0.18 ) net of the other variables. This is a large statistical effect size. However, in macro-level research having the correct causal model is tricky given so many correlated processes, therefore we ran alternative tests for the welfare state and GDP in M3 and M4. These models revealed much smaller effects: the explained variance does not increase as much as M2 and the betas are -0.23 and -0.19 respectively with the effect for GDP being not significantly different from zero. Finally, M5 includes all test variables and finds that disposable income inequality stands out as the only significant effect of all three, suggesting it is the variable most suited to explaining variance in risk perceptions. Looking at the fit statistics in the bottom two rows reveals that of M1-M5 the AIC is lowest in M2 and the log-likelihood is highest in M2 and M5. Together this information favors M2 as it is more parsimonious than M5, and based on the evidence is the model that is most likely to have produced the observed data. 
Table 1. Main Regression Analyses Testing the Impact of Income Inequality on Risk Perceptions

\begin{tabular}{|c|c|c|c|c|c|}
\hline Predictors & $\begin{array}{c}\text { M1 } \\
\text { Estimates }\end{array}$ & $\begin{array}{c}\text { M2 } \\
\text { Estimates }\end{array}$ & $\begin{array}{c}\text { M3 } \\
\text { Estimates }\end{array}$ & $\begin{array}{c}\text { M4 } \\
\text { Estimates }\end{array}$ & $\begin{array}{c}\text { M5 } \\
\text { Estimates }\end{array}$ \\
\hline Days Since Curve Inflection & $-0.00^{* *}$ & 0.00 & -0.00 & -0.00 & 0.00 \\
\hline New Case Rate & $0.18^{* * * *}$ & $0.20^{* * * *}$ & $0.18^{* * *}$ & $0.18^{* *}$ & $0.20^{* * * *}$ \\
\hline Government Intervention & -0.04 & -0.04 & -0.05 & -0.05 & -0.04 \\
\hline Disposable Income Inequality & & $0.03^{* * * *}$ & & & $0.03^{* * *}$ \\
\hline Welfare State Strength & & & $-0.08^{*}$ & & 0.04 \\
\hline GDP Per Capita & & & & -0.00 & -0.00 \\
\hline Observations & 74 & 74 & 74 & 74 & 74 \\
\hline $\mathrm{R}^{2} / \mathrm{R}^{2}$ adjusted & $0.238 / 0.206$ & $0.427 / 0.393$ & $0.277 / 0.235$ & $0.262 / 0.219$ & $0.433 / 0.382$ \\
\hline AIC & 47.802 & 28.783 & 45.915 & 47.492 & 31.982 \\
\hline log-Likelihood & -18.901 & -8.392 & -16.957 & -17.746 & -7.991 \\
\hline
\end{tabular}

Tbl 1 | Statistical models predicting risk perceptions in April, 2020 across 74 countries. Estimates are OLS regression coefficients.

We re-ran M2 in Table 1 using only the residuals of the dependent variable risk perceptions from M1 (see Technical Appendix). This provides the opportunity to visually inspect the impact of economic inequality on risk perceptions independent of outbreak severity and government intervention (see Fig 2). Essentially, it provides the most conservative estimate because income inequality is only statistically 'allowed' to explain the residuals, not part of the outbreak severity or government intervention effects. The result is that income inequality still has the same effect size and explains roughly $20 \%$ of the residual variance $\left(\mathrm{R}^{2}\right.$ $=0.203)$. What is particularly striking is that most of the highly equal countries in the lower left-hand corner of the graph have values below zero, meaning that their risk perceptions are lower than expected given the severity of the outbreak - here the 'Swedish Paradox' is very clear with Sweden being the furthest from zero despite a strong outbreak (see Fig 2). 


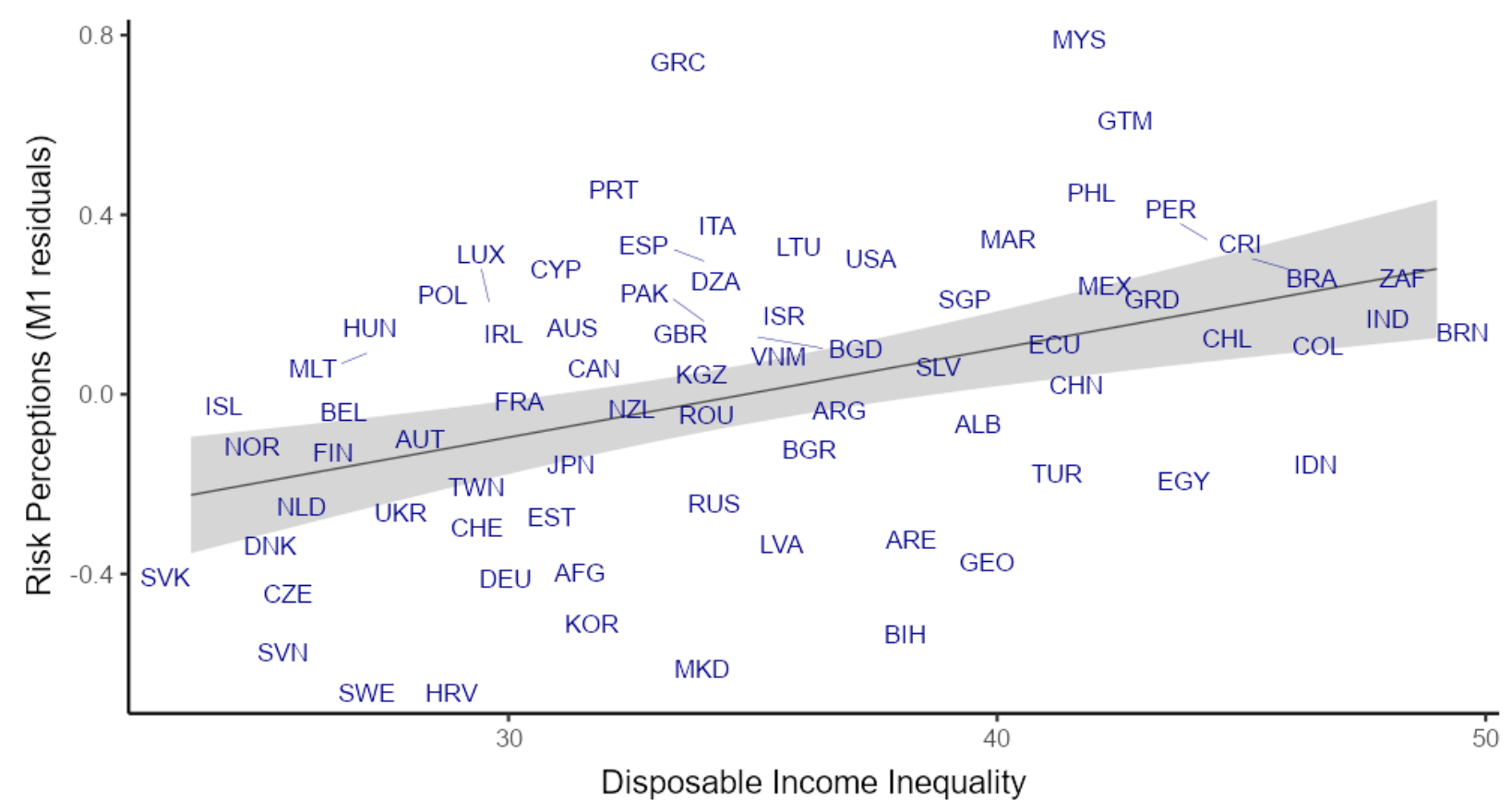

Fig 3 | Risk perception residuals explained by economic inequality. 'Over' and 'under' evaluation of the risk of the Coronavirus. Plotted residuals taken from M1 in Table 1. Interpretation of $y>0$ is 'over' concern, and $\mathrm{y}<0$ is 'under' concern compared to what we expect given the local outbreak severity and strength of government intervention.

Results for our alternative top 1\% income concentration measure are similar but suggest that top 1\% only explains about $9 \%$ of the unique variance of risk perceptions.

Income inequality and risk perceptions together predict greater infection increases

For our main analysis we used risk perceptions as both and independent and dependent variable and test for non-linear effects of risk perceptions and income inequality. Results in Table 2. The key aspects of these models are mediation of income inequality by risk perceptions. 


\begin{tabular}{|c|c|c|c|c|}
\hline $\begin{array}{c}\text { Infection Increase in May } \\
\text { (paths predicting } Y \text { ) }\end{array}$ & $\begin{array}{c}\text { M40 } \\
\text { Estimates }\end{array}$ & $\begin{array}{c}\text { M41 } \\
\text { Estimates }\end{array}$ & $\begin{array}{c}\text { M42 } \\
\text { Estimates }\end{array}$ & $\begin{array}{c}\text { M43 } \\
\text { Estimates }\end{array}$ \\
\hline Days Since Curve Inflection & $-0.03^{* * *}$ & $-0.03^{* * * *}$ & $-0.03^{* * *}$ & $-0.03^{* * * *}$ \\
\hline New Case Rate & $-0.22^{*}$ & $-0.18^{+}$ & -0.14 & -0.10 \\
\hline Government Intervention & $-0.13^{* *}$ & $-0.14^{* *}$ & $-0.10^{*}$ & $-0.10^{*}$ \\
\hline Risk Perceptions & $0.60^{* * * *}$ & $0.45^{* *}$ & $-10.87^{* * * *}$ & $-10.07^{* * *}$ \\
\hline Risk Perceptions^2 & & & $1.27^{* * *}$ & $1.18^{* * *}$ \\
\hline Disposable Income Inequality & & 0.02 & 0.02 & $-0.12+$ \\
\hline Disposable Income Inequality^ 2 & & & & $0.19^{+}$ \\
\hline $\begin{array}{l}\text { Risk Perceptions in April } \\
\text { (paths predicting M) }\end{array}$ & $\begin{array}{c}\text { M40 } \\
\text { Estimates }\end{array}$ & $\begin{array}{c}\text { M41 } \\
\text { Estimates }\end{array}$ & $\begin{array}{c}\text { M42 } \\
\text { Estimates }\end{array}$ & $\begin{array}{c}\text { M43 } \\
\text { Estimates }\end{array}$ \\
\hline Days Since Curve Inflection & $-0.00^{* *}$ & 0.00 & 0.00 & 0.00 \\
\hline New Case Rate & $0.19^{* * * *}$ & $0.20^{* * * *}$ & $0.20^{* * * *}$ & $0.20^{* * *}$ \\
\hline Government Intervention & -0.04 & -0.04 & -0.04 & -0.04 \\
\hline Disposable Income Inequality & & $0.03^{* * * *}$ & $0.03^{* * *}$ & $0.03^{* * *}$ \\
\hline Observations & 74 & 74 & 74 & 74 \\
\hline$R^{2} Y$ & 0.675 & 0.684 & 0.725 & 0.734 \\
\hline$R^{2} M$ & 0.238 & 0.427 & 0.427 & 0.427 \\
\hline AIC & 101.010 & 82.019 & 73.393 & 72.890 \\
\hline $\mathrm{BIC}$ & 133.267 & 118.884 & 112.562 & 114.364 \\
\hline log-Likelihood & -36.505 & -25.010 & -19.696 & -18.445 \\
\hline
\end{tabular}

Tbl 2 | Mediation models predicting risk perceptions and infection increase ratio, in 74 countries. Estimates are maximum-likelihood regression coefficients from a structural equation model. Maximum-likelihood estimation is employed because it can simultaneously estimate the interrelated coefficients from both equations predicting the two dependent variables $\mathrm{M}$ and $\mathrm{Y}$ (risk perceptions in April and infection increase in May)

M40 demonstrates the baseline predicted increase in infection from May $1^{\text {st }}$ to May $31^{\text {st }}$ of 2020 and the risk perceptions on average in April across 74 countries. This model explains roughly $68 \%$ of the variance in infection increase $(\mathrm{r} 2=0.675)$. Risk perception plays a key role in infection increase with a moderate sized effect that is unambiguously different from zero (beta $=0.24,95 \% \mathrm{CI}$ from 0.09 to 0.39 , see Technical Appendix). Logically this should indicate an indirect effect of economic inequality through risk perceptions because previously M5 revealed that economic inequality has a very large effect on risk perceptions. This is re-confirmed in M41 revealing the large effect of income inequality on risk perceptions and risk perceptions retaining a significant effect on infection increase, albeit slightly reduced when estimated as a simultaneous system of regressions (beta $=0.18,95 \% \mathrm{CI}$ from 0.01 to 0.35 ). The addition of income inequality to the model does not explain much more about infection increase ( 2 
change by only 0.009); however, it says a lot about risk perceptions ( $\mathrm{r} 2$ change of 0.189 , a roughly $80 \%$ increase in explained variance). M42 includes the squared term for risk perceptions and this has a noticeable impact on infection increase ( $\mathrm{r} 2$ change of 0.041 ) and when combined the risk perceptions and risk perceptions squared coefficients have a moderate effect (combined beta $=0.19,95 \% \mathrm{CI}$ not estimable as it is non-linear). Finally, M43 includes the squared term for income inequality. Results change only little (r2 increase of 0.009). M42 and M43 are nearly indistinguishable from a model fit perspective. This suggests that the main finding is the big linear effect of inequality on risk perceptions and the moderate curvilinear impact of risk perceptions on increase in infection; of course, after adjusting for covariates. Thus, we first report linear mediation effects in Fig 4, because most of the infection increase statistically speaking is a product of linear mediation.

\section{Mediation Effects}

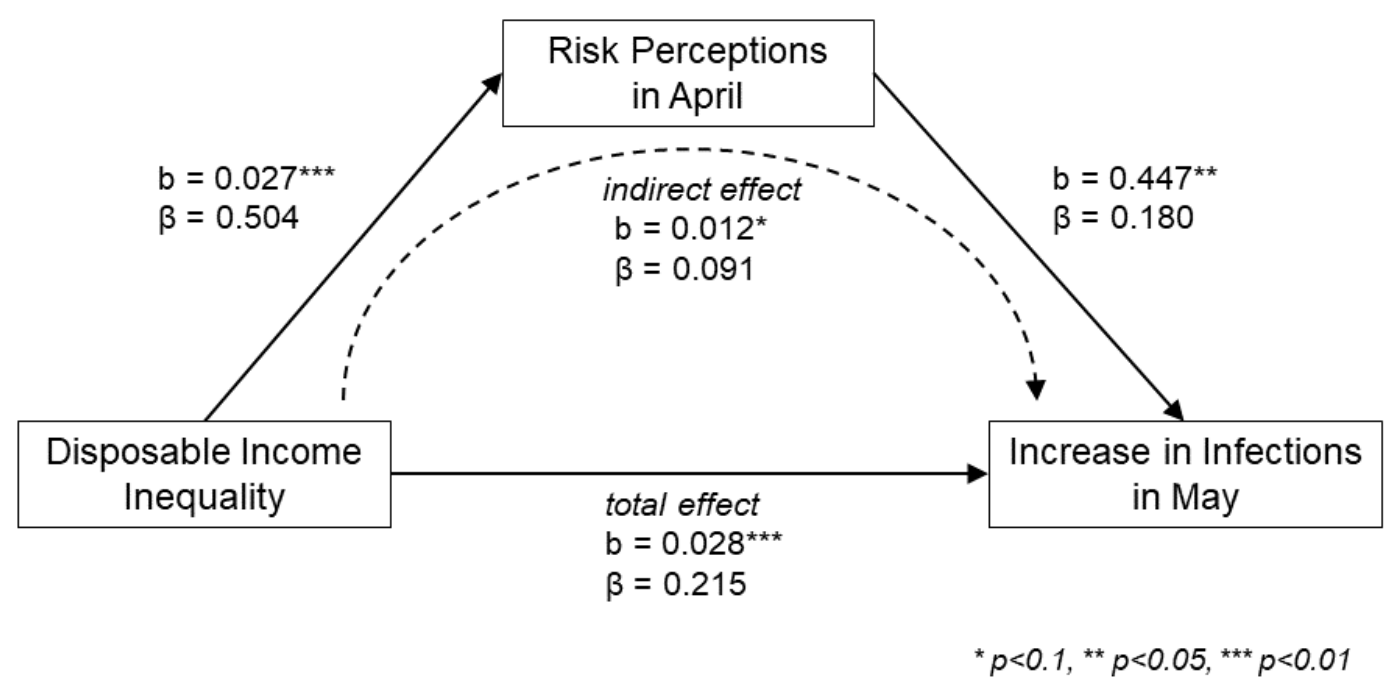

Fig 4 | Direct and indirect linear effects of economic inequality on infection rate in May, 2020. This mediation model taken from M41 in Tbl 2 demonstrates that income inequality has a moderate sized total effect (beta $=0.215)$ on the increase in infections in May after adjusting for the local outbreak severity and government intervention; however, $43 \%$ of this effect works indirectly through risk perceptions leaving inequality with split effects (direct beta $=0.122$, not significantly different from zero; indirect beta $=0.091$, significantly different from zero at $\mathrm{p}<0.10)$.

Despite a big linear effect, M42 and M43 reveal that there is slightly more to the story. Unfortunately, non-linear mediation makes a single calculation of direct and indirect effects impossible. Preacher and Hayes suggest reporting instantaneous rate of change using calculus for such case. This means that for 
any given value of income inequality there is a rate of change of the indirect effect. Fig 5 plots this instantaneous indirect effect (the goldenrod line). This effect must be interpreted as the change in indirect effect at any given value of inequality if inequality were to increase by one point from there. Thus, at any given negative value, any increase in inequality is predicted to decrease the infection rate in May, after adjusting for the severity of the outbreak locally and government intervention. Our results reveal that the rate of change flips directions at a Gini score around 31; however, in the realistic range of world Gini's this is rather low. Therefore, the potential impact of inequality is much greater toward highly unequal societies than in highly equal societies, and the rate of increase caused by any increase in inequality at Ginis above 40 is larger in absolute terms than at any point below 31.

We mention a few countries to aid in interpretation of Fig 5: For example in 2019, Sweden had a Gini of 26.3 falling in between Czechia (24.8) and Austria (27.5) among the most equal countries in our sample (and in the world); whereas Japan and Australia are close to 32, while highly unequal countries such as the United States and Albania are near 40 and extremely unequal societies such as South Africa and India are around 50.

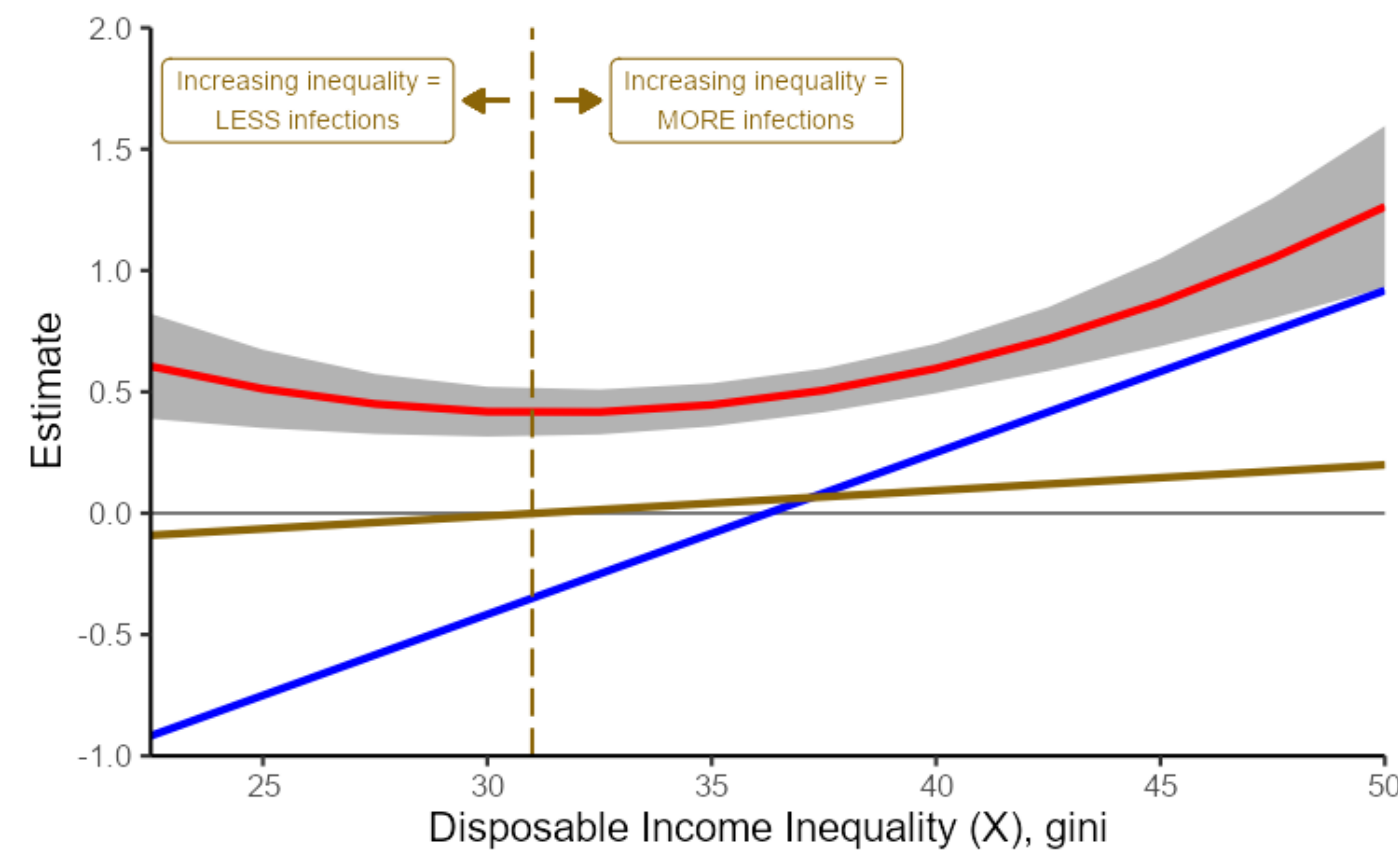

Infection Increase (Y)

- May 1st to May 31st ratio, predicted
Risk Perceptions (M)

April, 2020 predicted, centered
Instantaneous

Indirect Effect (rate of change)

Fig 5 | Instantaneous indirect effect of inequality through risk perceptions and predicted infection increase in May, 2020. The goldenrod colored line is the instantaneous 
indirect effect, indicating the rate of change of the indirect effect. Values left of the vertical dotted line indicate that increasing inequality at any given point would reduce deaths indirectly, while values to the right indicate the increasing inequality would increase deaths. The blue line is the predicted values of risk perceptions at each level of inequality (centered), and the red line is the ratio increase in infections in May, 2020 as a result of inequality and the predicted value of risk perceptions with all other variables held at their means.

\section{Robustness of the mediating effect}

Out of 2,000 plausible alternative distributions of the mean risk perception across countries, we find the robust mean slope (average of all 2,000) of the instantaneous indirect effect is 0.712 with a standard error of 0.009 and the robust intercept is 29.8 with a standard error of 0.118 . This means that our estimate of an inequality score of 31 as the inflection point of where the indirect effect switches from decreasing to increasing may be slightly overestimated (within 1.3 points). The robust mean slope suggests slight overestimation by about $30 \%$ ( 0.712 as opposed to our observed slope of 1.054$)$. Results are substantively the same with a non-linear indirect effect. This robustness check points out that the main findings here are that greater inequality is the main problem - the main statistical finding of this work - in explaining infection rates (18-day lead deaths).

\section{Conclusion}

We find a large statistical impact of disposable income inequality on risk perceptions in April and a moderate mediating role of disposable income inequality on increase in infections in May, measured with an 18-day lead of deaths from COVID-19. The bulk of our statistical explanation is linear: where inequality is higher, risk perceptions are higher and infection increase is higher as a result. However, we do find a significant albeit small curvilinear effect of inequality on increasing deaths in highly equal countries because risk perceptions are low, or at least lower than predicted based on the severity of the outbreak and the degree of government intervention.

A critical limitation of this study is the nature of risk perceived by the public in the survey and the resulting behaviors, both of which are unobserved in the CiS data. The CiS questions give excellent face validity regarding "concern" over the negative effects of the "Coronavirus", but there are no specifics. The perceived risks could be health related, as individuals worry about their own risk of infection or perhaps the infection of a close friend or family member. The risk perceptions could also be economic as the pandemic had a tremendous impact on unemployment and financial security, especially of the majority of middle class, blue collar and working poor individuals and their families who rarely have 
savings. The risk could be quite subjective, like fear of losing one's personal freedoms or ability to travel for pleasure. Although the $\mathrm{CiS}$ data are a fantastic open science tool that was rapidly organized right at the beginning of the pandemic, we cannot disentangle the exact nature of risk. We suspect that risk perceptions in any form at the extremes lead to behaviors that risk infection increases, but these behaviors are also unobserved. Without more detailed information it is difficult to translate these findings into policy or behavioral recommendations, other than risk perceptions at the extremes are a threat to societies in a pandemic.

Other potential limitations are the sample and sample size. The sample is entirely an internet survey; this leads to potential bias as it is not likely a random sample of the entire populations of these countries. In fairness it is the only possible mode given that face-to-face surveys were not safe to conduct at the time and organizing a face-to-face survey in such a short time to capture the height of the first outbreak wave would have been impossible. If anything the CiS is likely to underrepresent older aged persons, these are the persons who are most at risk of death. Therefore, it may under-estimate the actual risk perceptions by country, but whether this varies by country we cannot say. The sample size is also concerning because the nature of countries is highly complex legally, politically, socially, culturally, economically and demographically. We cannot possibly account for all these factors with a sample of 74 countries. In terms of measures of central tendency, we are already pushing the limits as M43, our preferred model, has less than 10 cases per independent variable. The complexities of countries and the local outbreak are reduced down to just three independent variables and one test variable of economic inequality.

Regarding the 'Swedish paradox' of extremely high per capita deaths but very low risk perceptions, we are only partially successful in explaining it. We suspect that longitudinal data and more detailed analyses of the social security system and the cultural norms would reveal more about risk perceptions in Sweden and the other highly equal societies of the world. Nonetheless we propose that a Gini around 31 is the tipping point in risk perceptions, and further research should test if this is a robust value and what it is about this value that causes a shift toward worse outcomes at the hands of a pandemic. We remind the reader that income inequality itself is more of an indicator than a direct cause as it is associated with myriad factors such as the degree of redistribution of social and tax policy, the amount of group oppression (along racial, ethnic and cultural lines) and social norms of justice and ideology. Therefore, we conclude that inequality can lead to more deaths in a pandemic but cannot isolate exactly why, other than what is already known in terms of crowded dwellings, work conditions and neighborhoods and issues of public health information and access. Here we add the important role of risk perceptions to this list. 


\section{References}

1. Erev I, Plonsky O, Roth Y. Complacency, panic, and the value of gentle rule enforcement in addressing pandemics. Nat Hum Behav. 2020 Aug 14;1-3.

2. Alvaredo F, Chancel L, Piketty T, Saez E, Zucman G. World Inequality Report 2018. Belknap Press; 2018.

3. Brady D, Finnigan RM, Hübgen S. Rethinking the Risks of Poverty: A Framework for Analyzing Prevalences and Penalties. Am J Sociol. 2017 Nov 1;123(3):740-86.

4. Van de Werfhorst HG, Salverda W. Consequences of economic inequality: Introduction to a special issue. Res Soc Stratif Mobil. 2012 Dec 1;30(4):377-87.

5. Wilkinson R, Pickett K. Income Inequality and Social Dysfunction. Annu Rev Sociol. 2009;35:493511.

6. Jachimowicz JM, Szaszi B, Lukas M, Smerdon D, Prabhu J, Weber EU. Higher economic inequality intensifies the financial hardship of people living in poverty by fraying the community buffer. Nat Hum Behav. 2020 Jul;4(7):702-12.

7. Buttrick NR, Oishi S. The psychological consequences of income inequality. Soc Personal Psychol Compass. 2017;11(3):e12304.

8. Fairbrother M, Martin IW. Does inequality erode social trust? Results from multilevel models of US states and counties. Soc Sci Res. 2013 Mar 1;42(2):347-60.

9. Ahmed F, Ahmed N, Pissarides C, Stiglitz J. Why inequality could spread COVID-19. Lancet Public Health. 2020 May 1;5(5):e240.

10. Rutter PD, Mytton OT, Mak M, Donaldson LJ. Socio-economic disparities in mortality due to pandemic influenza in England. Int J Public Health. 2012 Aug 1;57(4):745-50.

11. Elgar FJ, Stefaniak A, Wohl MJA. Research paper The trouble with trust: Time-series analysis of social capital, income inequality, and COVID-19 deaths in 84 countries. Soc Sci Med. 2020 Sep $16 ; 113365$.

12. Banik A, Nag T, Chowdhury SR, Chatterjee R. Why Do COVID-19 Fatality Rates Differ Across Countries? An Explorative Cross-country Study Based on Select Indicators. Glob Bus Rev. 2020 Jun 1;21(3):607-25.

13. Migone AR. The influence of national policy characteristics on COVID-19 containment policies: a comparative analysis. Policy Des Pract. 2020 Aug 18;0(0):1-18.

14. Balmford B, Annan JD, Hargreaves JC, Altoè M, Bateman IJ. Cross-Country Comparisons of Covid-19: Policy, Politics and the Price of Life. Environ Resour Econ. 2020 Aug 1;76(4):525-51.

15. Taylor-Gooby P, Zinn JO. Current Directions in Risk Research: New Developments in Psychology and Sociology. Risk Anal. 2006;26(2):397-411. 
16. Soroka SN, Wlezien C. Degrees of Democracy: Politics, Public Opinion and Policy. Cambridge: Cambridge University Press; 2010.

17. Lieberoth A, Ćepulić D-B, Rasmussen J, Travaglino GA, Tuominen J, Tran T. COVIDiSTRESS Global Survey. Open Sci Framew Osfioz39us. 2020;

18. Breznau N. The Welfare State and Risk Perceptions: The Novel Coronavirus Pandemic and Public Concern in 70 Countries. Eur Soc. 2020 Aug 1;0(0):1-14.

19. Steinmetz H, Schmidt P, Tina-Booh A, Wieczorek S, Schwartz SH. Testing measurement invariance using multigroup CFA: differences between educational groups in human values measurement. Qual Quant. 2008;43(4):599-616.

20. Verity R, Okell LC, Dorigatti I, Winskill P, Whittaker C, Imai N, et al. Estimates of the severity of coronavirus disease 2019: a model-based analysis. Lancet Infect Dis. 2020 Jun 1;20(6):669-77.

21. Naumann E, Mata J, Reifenscheid M, Möhring K, Wenz A, Rettig T, et al. Die Mannheimer Corona-Studie: Schwerpunktbericht zum Angstempfinden in der Bevölkerung [Internet]. University of Mannheim, Germany; 2020. Available from: https://www.unimannheim.de/media/Einrichtungen/gip/Corona_Studie/Schwerpunktbericht_Angstempfinden_Mann heimer_Corona_Studie.pdf

22. Dong E, Du H, Gardner L. An interactive web-based dashboard to track COVID-19 in real time. Lancet Infect Dis. 2020 May 1;20(5):533-4.

23. Hale T, Webster S, Petherick A, Phillips T, Kira B. Oxford COVID-19 Government Response Tracker. Oxford University: Blavatnik School of Government; 2020.

24. Roser M, Hasell J, Ortiz-Ospina E, Ritchie H. Coronavirus Pandemic (COVID-19): Policy Responses to the Coronavirus Pandemic. Our World Data [Internet]. 2020; Available from: https://ourworldindata.org/coronavirus

25. Solt F. The Standardized World Income Inequality Database. Soc Sci Q. 2016;97(5):1267-1281.

26. Bollen KA. Total, Direct, and Indirect Effects in Structural Equation Models. Sociol Methodol. 1987;17:37-69.

27. Hayes AF, Preacher KJ. Quantifying and Testing Indirect Effects in Simple Mediation Models When the Constituent Paths Are Nonlinear. Multivar Behav Res. 2010 Aug 6;45(4):627-60. 